\title{
Teoría y praxis en las instituciones educativas 1
}

...no se debe caer en el espejismo de pensar que un cambio de ideas es un cambio de realidad o que un cambio de intención - la pureza de intención-basta pare cambiar la realidad... es necesario emprender una praxis transformadora de la realidad social.

Ellacuria

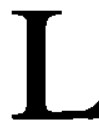

a reflexión sobre "Teoría y praxis en las instituciones educativas" constituye una continuación del tema presentado en el II Encuentro Mesoamericano de filosofía titulado: "Mundialización y Liberación" realizado en Nicaragua en diciembre de 1995. En este encuentro destacábamos que las instituciones educativas universitarias desempeñan una función política importante en la sociedad. Se trata de contribuir a la transformación de las estructuras injustas, desde su propia especificidad, mediante la investigación, la docencia y la proyección social ${ }^{2}$. El énfasis en esta ponencia se ponía en el aporte que las instiluciones superiores pueden dar a la transformación del país, a través de los diagnósticos científicos sobre la realidad sociopolítica y las propuestas de solución con base en tales diagnósticos.

Lo que pretendemos con esta breve reflexión es mostrar la necesidad de que las teorías que las instituciones educativas plantean para las instituciones sociales en general, en orden a la construcción de una sociedad más justa y democrática, se pongan en práctica en el interior de dichas instituciones. Esto es de vital importancia - si las escuelas, colegios y universidades - quieren desempeñar eficazmente y con credibilidad su misión social.

Queremos aclarar que estas líneas que presentaremos en seguida son el resultado de la experiencia de ocho años de labor docente, experiencias compartidas con maestros, del sentir de muchos de mis alumnos(as) y de los acontecimientos 
que se han realizado, en esta década, en la historia de El Salvador. Estos hechos presentan grandes desafíos para las instituciones educativas si quieren responder efectivamente a la función que les compete.

En primer lugar, la finalización del conflicto armado mediante la firma de los acuerdos de paz el 16 de enero de 1992. Según el documento final, los acuerdos de paz buscan "impulsar la democratización del país, garantizar el irrestricto respeto a los derechos humanos y reunificar a la sociedad salvadore-

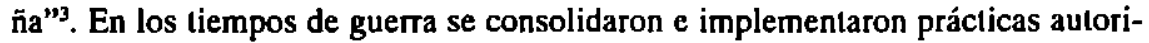
tarias en el ejercicio del poder en todos los ámbitos de la sociedad, es decir, en lo económico, político, social y familiar. Los acuerdos pretenden superar la concepción verticalista del ejercicio del poder, estableciendo mecanismos más participativos o democráticos en la toma de decisiones.

En segundo lugar, la Reforma Educativa implementada por el Ministerio de Educación. En el informe presentado por la Comisión de Educación, Ciencia y Desarrollo se afirma que el sistema político configuró el perfil de la educación. Esto implica que el autoritarismo propio del sistema político se reprodujo en el sistema educativo. Ante esta realidad, se plantea la necesidad de democratizar la educación para contribuir a la democratización de la sociedad salvadoreña.

Ambos aconlecimientos apuestan por una democratización de la sociedad y de la educación respectivamente. Planteados estos aspectos fundamentales, explicaremos brevemente los dos apartados que constiluirán esta reflexión: en primer lugar, las relaciones entre teoría y praxis; en segundo lugar, la teoría y la praxis en las instituciones educativas.

\section{Relación entre teoría y praxis}

En la historia de la filosofía se ha concebido de diversas formas el vínculo existente entre teoría y praxis. En algunos momentos de la historia, la actividad teórica adquirió un rango de superioridad con respecto a las actividades prácticas o productivas. Las actividades teóricas eran consideradas como propias de los hombres libres, y las actividades productivas de los esclavos. Esta forma de concebir las relaciones entre teoría y praxis la encontramos en la cultura griega. Para Platón y Aristóteles, por ejemplo, tiene prioridad la teoría sobre la praxis. Para Platón "la vida teórica, como contemplación de las esencias, la vida contemplativa adquiere una primacía y un estatuto metafísico (...) Vivir propiamente es contemplar"'. El ser humano se realiza en la contemplación de las ideas etemas, inmutables, es decir, liberándose de los obstáculos del mundo sensible y corpóreo que impiden la contemplación de las esencias inmutables. Platón reconoce que la teoría debe ser práclica, es decir, mantenerse en unidad, pero solamente en el ámbito polílico, aunque la teoría sigue siendo la prioritaria. 
De ahí que plantee que el más indicado para dirigir la polis sea el filósofo porque es el que conoce la verdadera realidad. Sin embargo, el trabajo humano productivo es considerado de rango inferior. Aristóteles desprecia lambién las actividades productivas y prioriza las actividades teóricas. Esto se fundamenta en la concepción de "hombre como ente de razón, la teoría se basta a sí misma, sin necesidad de ser aplicada o de subominarse a la práctica"s. Sin embargo, al igual que platón, plantea que la teoría puede aplicarse a la política, aunque no pretende, según lo planteado por Sánchez Vázquez, que la praxis política se ajuste a principios teóricos absolutos.

En el renacimiento y la modernidad, la relación entre leoría y praxis sufre un cambio esencial. La teoría no es concebida como un saber desinteresado o como fin en sí misma, sino como un saber que busca transformar la realidad para el beneficio del ser humano. Desde esta perspectiva, se reduce la brecha existente entre las actividades teóricas y las aclividades productivas; ya que se valorizan las acciones del ser humano y no sólo la teoría, aunque sigue teniendo el primer puesto. Como diría Francis Bacon, "el conocimiento es poder". Es decir, es necesario conocer para transformar la realidad para bien de la humanidad. Las actividades prácticas ya no se conciben aquí como algo humillante, si no como el medio para dominar y cambiar la naturaleza .

Posteriormente otros economistas clásicos "se caracterizarán por la exaltación de la praxis material productiva... y elevan a la categoría de proxis en general . A ellos corresponde el mérito de haber visto en el trabajo humano la fuente de toda riqueza y de todo valor"'. Marx cuestionará las insuficiencias y limitaciones de estos planteamientos, por reducirse sólo al ámbito económico. La praxis ocupará el primer lugar y la teoría se convertirá en un instrumento de la misma. La filosofía como teoría no tiene que ser un medio legitimador de las estructuras reales, sino que el medio de su transformación.

En las tesis sobre Feuerbach, Marx criticar a los filósofos que se han dedicado solamente a la interpretación de la realidad. En la tesis XI afirma: "Los filósofos no han hecho más que interpretar de diversos modos cl mundo, pero de lo que se trata es de trasformarlo"'. Desde esta perspectiva, la praxis persigue la transformación de la naturaleza y las estrucluras injustas de la sociedad. De ahí que Marx no compartía las posturas de algunos filósofos neohegelianos que consideran que cuestionando la realidad se posibilitaba la transformación de las estrucluras de la misma. Es decir, se confiaba excesivamente en el poder de las ideas como medio de transformación de la realidad. Se concibe, desde esta perspectiva, el carácter práctico de la teoría filosófica. Se trata de que "la función práctica de la filosofía adoptaba la forma de una crítica de los elementos irracionales de la realidad, crítica incesante y a fondo de las instituciones petrificadas..." Se creía que mediante la crílica filosófica se podía transformar las irracionalidades presentes en el mundo real. Pero la realidad mostraba lo contra- 
rio, por más cuestionamientos que se realizaran, las estructuras de la realidad seguían manteniéndose iguales. Estos planteamientos según Marx eran insuficientes. Era necesario emprender una praxis real si se quería lograr la transformación de la realidad.

La postura ellacuriana en tomo a la relación entre teoría y praxis se expresa mediante la afirmación de que las solas ideas no transforman la realidad, sino que es importante emprender una praxis, que haga posible transformar las estructuras injustas en favor de los sectores desposeídos de la sociedad. Ellacuría planteaba que sería una ingenuidad de nuestra parte, el pensar que cambiando las ideas se transforma la realidad. Estas afirmaciones se comprenden mejor si se considera que en la realidad salvadoreña, centroamericana y latinoamericana predominaba y siguen predominando las condiciones de miseria y empobrecimiento causadas por la injusticia estructural. Ante esto califica de superficial la pretensión de algunos filósofos que piensan que al interpretar y analizar críticamente la realidad la transforman. En Ellacuría encontramos que la teoría y la praxis constituyen una unidad estructural. Esto lo podemos corroborar en el siguiente texto: “...las formulaciones teóricas, también las filosóficas, tienen un puesto indispensable y necesario tanto a la hora del análisis y de la interpretación, a la hora de la valoración y de la justificación, como a la hora de la acción y de la transformación. La pura praxis no existe y cuando se pretende conslituirla en la única fuente de luz no sólo es una praxis ciega, sino que además es obcecada"y. Aquí se destaca el papel fundamental de la teoría como momento necesario de toda praxis, que tiene como propósito la transformación de las estructuras de injusticia en el ámbito económico y del autoritarismo en el ámbito político, educativo, etc. La praxis, según nuestro autor, está referida a la "totalidad del proceso social en cuanto transformador de la realidad tanto natural como histórica"'t. Esta praxis, que posee un momento teórico, en el sentido que puede orientarla, pretende lograr la construcción de unas estrucluras sociales que posibiliten a los seres humanos, de una sociedad concreta, vivir en unas condiciones más dignas.

En definitiva, pues, para Ellacuría cambiar las ideas no garantiza que se transformen las condiciones estructurales de injusticia y miseria, es necesario que las fuerzas de la historia asuman esas ideas de tal manera que puedan emprender una praxis liberadora . Dentro de las fuerzas de la historia se encuentran, según Ellacuría, las instituciones sociales, como los partidos políticos, Iglesia, militares, etc. En estas instituciones se constata el hecho de que la teoría o " discursos conceptuales en nada se adecua a su práctica colidiana, aunque se supone, cuando no hay patente hipocresía, que aquel discurso se mantiene honradamente"'l. En otras palabras, existe una contradicción entre lo que afirman en el nivel teórico y lo que hacen en la práctica. Este es precisamente la reflexión que a continuación presentaremos de las instituciones educativas. En estos momentos de cambios a nivel mundial y regional es importante que las diversas 
instituciones se autoevalúen si están desempeñando eficazmente y con credibilidad su función en la sociedad. Esto es más urgente para las instituciones educativas, cuya misión social es de vital importancia en estos momentos de crisis en los que vivimos.

\section{Principales tendencias entre el discurso conceptual y la práctica real en las instituciones educativas}

En el apartado anterior planteamos, brevemente, las tendencias que se han dado en cuanto a las relaciones entre teoría y praxis. Estas formas de concebir las relaciones entre leoría y praxis han hecho eco en nuestros pueblos y han conducido a planteamientos como, por una parte, se ha considerado que lo más fundamental son las actividades teóricas, esto más que todo en algunas inslituciones religiosas, educativas, etc. Desde este punto de vista se ha minusvalorado a la praxis. Por otra parte, se le ha dado el papel fundamental a la praxis en detrimento de la teoría. Lo importante es la praxis, ya que posibilita la transformación de las estructuras de la realidad social. Ambos casos son extremos y no hacen justicia al papel que tanto teoría y praxis juegan en la interpretación y transformación de la realidad. La concepción que asumiremos en esta breve reflexión es la planteada por Ignacio Ellacuría. Ni las ideas ni la praxis separadas, una de la otra, pueden contribuir a transformar la realidad. Esta postura es la que consideramos más acertada para esbozar algunas tendencias que hemos detectado en cuanto a la relación entre el discurso pedagógico y/o político y la praxis intema en las instituciones educativas en nuestro país.

En cuanto al discurso pedagógico se insiste en superar la relaciones verticales y autoritarias entre alumnos y profesores, de tal manera que posibiliten establecer unas relaciones más humanas y más armoniosas. Por otra parte, el empleo de metodologías más participativas para superar la concepción de educación bancaria y que haga posible una educación que fomente la creatividad, la solidaridad, la cooperación, la libertad, etc. ${ }^{12}$

En cuanto al discurso sociopolílico, de algunas instiluciones educalivas ${ }^{13}$ de nivel superior, se puede catalogar como un discurso de avanzada o liberador. Es decir, mediante sus análisis científicos y/o filosóficos sobre la realidad social, denuncian las violaciones a los derechos humanos, la injusticia estruclural, la cornupción, el autoritarismo y proponen, en algunos de los casos, ciertas soluciones como la necesidad de construir una sociedad democrática y más justa en donde se respeten los derechos humanos, donde exista transparencia y participación en el ejercicio del poder. No cabe duda que es necesario el cambio de concepciones pedagógicas tradicionales y, a la vez, de la necesidad de una producción teórica que desenmascare los mecanismos ideologizantes que legitiman las injusticias a las que están sometidos grandes sectores de nuestras sociedades. 
Pero, según lo planteado en el primer apartado, es necesario llevar a la práctica esas ideas para ser coherente con los discursos que se pronuncian y poder generar el cambio que realmente se espera, y poder cumplir así adecuadamente la misión sociopolítica que les compete.

Eslo nos lleva a preguntarnos, ila praxis intema de las instituciones educativas es coherente con las concepciones teóricas que sustentan? ¿Las concepciones teóricas sustentadas y sus prácticas realizadas son adecuadas para contribuir a la construcción de una sociedad más jusla y democrática? Para aproximamos a estas respuestas plantearemos tres tendencias en las que se pueden ubicar a nivel general las instiluciones educativas:

En primer lugar, nos encontramos con instituciones cuyos concepciones pedagógicas, sociopolíticas y sus prácticas son tradicionales o conservadoras. Esto se expresa en el tipo de relaciones que se establecen entre alumnos, profesores y directores o autoridades de las escuelas, colegios y universidades. Se trata de unas relaciones jerárquicas, verticales, autoritarias que se establecen en los tres componentes fundamentales de las instituciones.

En el caso de las escuelas y colegios, el alumno liene que aceplar acríticamente y de manera sumisa las normas, ideas, etc. que le impone el maestro. Por otra parte, el profesor tiene que mantener una actitud similar ante órdenes y disposiciones inconsultas que le impone el jefe inmediato o el director (a). Al direclor(a) se le debe rendir obediencia incondicional, porque de lo contrario, en el caso de los alumnos pueden ser sancionados o expulsados y los maestros amonestados o despedidos. Cuando un estudiante, por ejemplo, se atreve a cuestionar al profesor, se le estereotipa y cataloga de rebelde.

De la misma forma, si un profesor asume una actitud de criticidad con respecto a las disposiciones de la autoridad máxima del centro educativo, se le margina. En este tipo de instiluciones se ve con buenos ojos a los alumnos, profesores sumisos y obedientes, es decir, que no se oponen y están de acuerdo en todo lo que dice la autoridad. Estos alumnos tienen la posibilidad de ser condecorados o darles premios al final del año escolar por haber observado buena conducta. Estos profesores son los que tienen mayores garantías de mantener su trabajo en dichas instituciones y mayores posibilidades de ascender a algún puesto de dirección en los centros educativos, porque garantizarán fidelidad al superior y la reproducción del sistema.

En las universidades de esta tendencia se da una variante por sus específicas funciones de docencia, investigación y proyección social que debe desempeñar. Se establecen relaciones verticales enire alumnos, profesores y autoridades de manera más sofislicada, en algunos casos, abiertamente en otros. Lo problemático en estas instiluciones está en adoptar una aclitud profesionalizante, es decir, que forman profesionales para que inlegren y reproduzcan las filas del sistema 
injusto cuyos valores reproducen. No realizan investigaciones que posibiliten analizar científicamente la realidad natural e histórica, lo cual les impide que se propongan alternativas de solución a los graves problemas en los que encuentran sometidos grandes sectores de nuestro país. Esto lleva a que este tipo de instiluciones universitarias mantengan una actitud de sumisión y acriticidad ante los poderes dominantes, siempre $y$ cuando no afecten sus intereses y privilegios de los cuales gozan como instituciones educativas.

En definitiva, las instituciones educativas con esta tendencia tratan de justificar el estado de cosas vigente que le es favorable, lo que conlleva al establecimiento de un tipo de relaciones autoritarias entre los integrantes de dichas instiluciones. Estas instituciones todavía no han entendido que a nivel pedagógico y político se están produciendo cambios que tienen que ir asumiendo para responder a su misión sociopolítica, en favor de una transformación de las estructuras generadoras de marginación económica, política, cultural, etc.

En la segunda tendencia nos encontramos con teorías pedagógicas y discursos políticos progresistas o liberadoras y sus prácticas internas reales tradicionales y conservadoras. Se trata de una incoherencia entre lo que se profesa en teoría y lo que se hace realmente en la práctica. Esta tendencia se observa tanto en escuelas y colegios como también en universidades. Desde esla perspectiva, existe claridad de que como instituciones educativas deben contribuir a la transformación de las estructuras que impiden que las mayorías populares puedan vivir en unas condiciones más dignas. El discurso pedagógico y político expresa esa convicción.

En cuanlo a algunos colegios de educación básica y media, las nuevas ideas pedagógicas han hecho eco. Si analizamos sus objetivos nos damos cuenta que buscan la formación de "nuevos hombres y mujeres para una sociedad nueva". Esto significa la formación de un ser humano libre de las ataduras que nos presenta la sociedad; con capacidad crítica anle las valores que predominan en la sociedad: consumismo, la avaricia, odio, egoísmo, etc. Se Irata de educar en y para la liberlad. Estos planteamientos se enfatizan en las capacitaciones que unos colegios capitalinos imparten a sus maestros al inicio del año y se insiste en la implementación de metodologías más participativas que vayan posibilitando el ejercicio de la creatividad y libertad en los estudiantes. Sin lugar a dudas, este tipo de educación necesita de un tipo distinto de maestro. El maestro facilitador y amigo de los estudiantes. Implica una nueva forma de concebir la autoridad del maestro y la del director o directora. Ahora bien, ¿qué sucede en la praxis educativa colidiana? La organización jerárquica interna de las instiluciones impiden, en muchos de los casos, el ejercicio de la creatividad, libertad y criticidad de estudiantes, docentes y administrativos. Se siguen implementando prácticas autoritarias, verticalistas e inconsultas. Se hace lo que dice el profesor, el coordinador de disciplina y el director(a). Aunque los argumentos planteados, 
ante una situación concreta, por algún miembro de la comunidad educativa sea razonable. Por otro lado, muchos estudiantes y docentes manifiestan miedo a expresar sus razones por las represalias a las que puedan ser sometidos por los que ejercen la autoridad. Esto manifiesta la brecha existente entre lo que se afima (teoría) y lo que realmente se hace (praxis).

Por otra parte, las universidades desempeñan una función política fundamental en la sociedad, tal como lo planteábamos en el Segundo Encuentro en Nicaragua. Ante las irracionalidades, manifestadas en la injusticia estructural de nuestras sociedades, la universidad no se puede quedar sorda ni muda en cuanto cultivadora de la razón científica y filosófica. Como diría José María Tojeira, la universidad "tiene que buscar y ponerse al servicio de la verdad... para lograr que la vida en nuestra región sea más humana y por tanto más digna". Por otra parte, a nivel mundial, la universidad debe "preocuparse por los fenómenos de imperialismo que permanecen tras los procesos de globalización y mundialización"'14. Según dicho aulor, si una universidad, en nuestros contextos, no es antiimperialista ya sea por interés, miedo, etc. no podría considerarse universidad. Estos planleamientos destacan la misión política de la universidad para con la sociedad. Esto es fundamental para toda universidad que quiera cumplir con efectividad su misión política. Estamos ante la presencia de unos planteamientos que buscan contribuir a la superación de las condiciones de inhumanidad en la que viven sometidas unas grandes mayorías de seres humanos en nuestros pueblos.

Ahora bien, la inquietud que nos surge ante universidades que han desempeñado una función beligerante en la sociedad y han denunciado los atropellos a los derechos de las personas, las injusticias cometidas en el ámbito laboral, la corrupción de las instituciones del Estado, etc. y han exigido transparencia en el ejercicio del poder, la superación del autoritarismo y construir una sociedad democrática, estas instituciones, ¿son coherentes y practican internamente lo que sostienen a nivel teórico? Al observar de cerca el funcionamiento real de algunas instituciones con discursos progresistas o liberadores se puede constatar que existen algunas contradicciones entre lo que afirman a nivel teórico y lo que en la realidad hacen. Lo que predomina en muchos aspectos son prácticas autoritarias, no hay mayores espacios de participación de los miembros que la integran. Se siguen dando las relaciones verticales entre alumnos, maestros y autoridades.

Esta dualidad manifiesta que existe conciencia de los retos fundamentales que tienen las instituciones educativas para con la sociedad, pero "sus prácticas siguen apegadas a una tradición autoritaria y conservadora que no permite verdaderos avances tendientes a transformar radicalmente las instituciones educativas para ponerlas a tono con el nuevo conlexto de la sociedad contemporánea"ls. Estamos ante unos planteamientos téricos que consideramos necesarios y adecuados ante la irracionalidad reinante en nuestros pueblos, pero que la práctica 
interna contradice lo que afirma teóricamente. ¿Será posible y creíble contribuir al cambio de las estructuras injustas y a la democratización de un país, si las instituciones que pregonan y exigen dichos cambios en teoría no practican internamente dicha teoría? El cambio de un país pasa por el cambio de sus instituciones, si éstas no cambian y si no practican intemamente lo que sostienen, no puede haber un cambio real en nuestras sociedades. No es posible que un país cambie realmente las estructuras injustas y autoritarias si no cambian a nivel interno las instituciones que forman dicho país.

La solución a los problemas que sustentan las tendencias anteriores estaría en la tercera tendencia, que se puede plantear en términos de una concepción liberadora y una práctica liberadora. Esta tendencia es implementada, en instituciones con las tendencias anteriores, por algunos maestros(as) en sus clases y por algunos maestros que desempeñan cierto rango de autoridad en las estructuras jerárquicas. Algunos docentes han asumido el reto de poner en práctica las ideas pedagógicas y sociopolíticas que buscan transformar las esiructuras autoritarias y contribuir a formar un tipo de ser humano solidario con los más desprotegidos de la sociedad. Esta tendencia, si la tomamos en serio, posibilitaría la superación de la relación autoritaria entre alumnos, maestros autoridades y se establecerían una relaciones más libres, democráticas y participativas. No se trataría de eliminar a la autoridad, porque entonces tendríamos anarquía, si no de transformar radicalmente su sentido en beneficio de la consolidación de relaciones más humanas entre los integrantes de las instituciones educativas, superando así las relaciones prepotentes y absolutistas.

Si queremos que las teorías pedagógicas y sociopolíticas liberadoras se realicen efectivamente en nuestras instituciones educativas, es necesario transformar las relaciones internas de poder existentes en las instituciones educativas. Es necesario llevar a la práctica real y efectivamente las teorías tendientes a humanizar las relaciones entre los integrantes de las instituciones educativas y las que buscan superar las relaciones autoritarias y sumisas que predominan en muchas instituciones educativas salvadoreñas.

Quisiera concluir estas líneas, citando a Cajiao e invilando a la reflexión a profesores, dirigentes de las instituciones educalivas y a los responsables de la educación en nuestros países, "que no basta consagrarse a enunciar cuidadosamente los propósitos de transformación del sistema educativo y de nuestros paises, ni enunciar detalladamente las metas del aprendizaje, ni conocer profundamente las teorías que apuntan a modificar los sistemas de aprendizaje y construcción del conocimiento. Todo esto es necesario pero no suficiente (...) Es indispensable tomar conciencia del enorme peso de la tradición autoritaria en la cual han sido y hemos sido formados educadores y administradores. Si se quiere cumplir eficazmente y con credibilidad la misión sociopolíica es necesario transformar las formas de organización basadas en determinados mecanismos de po- 
der autoritario, para que las nuevas ideas pedagógicas y sociopoliticas que se generan puedan ser una realidad $y$ no se queden en puras teorias ${ }^{16}, y$ para contribuir eficaz y con credibilidad a la construcción de una sociedad democrática y justa.

\section{Notas}

1. Ayala Perdomo, E. O. "La función política de la universidad en la sociedad actual", Mundialización y liberación, Ensayos filosólicos, II Encuentro Mesoamericano de filosofía, Colección pensamiento, Managua, 1996.

2. Carr, W. Una teoría para la educación. Hacia una investigación educativa crítica, Ediciones Morata, Madrid, 1996.

3. Cajiao, F. Poder y justicia en la Escuela colombiana, Editores FES, Colombia, 1995.

4. Colorn, A. J. y Mélich, J. C. Después de la modernidad. Nuevas filosofias de la educación, Ediciones paidós, España, 1994.

5. Ellacuría, I. "Función liberadora de la filosolía", ECA, No 435-436, San Salvador, 1985.

6. Marx, K. Los manuscritos de 1844 y las tesis sobre Feuerbac, UCA Editores, San Salvador, 1987.

7. Sánchez Vázquez, A. Filosofía de la praxis, Editorial Grijalvo, Tercera Edición, México, 1973.

8. Tojeira, J. M. "La universidad y el servicio de la verdad en Centroamérica", Realidad, No 54, San Salvador, 1996.

\section{Documentos Especiales}

9. Acuerdos de Paz, Documento especial, ECA, No 519-520, San Salvador, 1992.

10. Transformar la educación para la paz y el desarrollo de El Salvador, Documento especial, Comisión Nacional de Educación, Ciencia y Desarrollo, ECA, No 561562, San Salvador, 1995.

11. La educación en El Salvador, Número monográfico, ECA, No 547-548, San Salvador, 1994.

12. "Volver a pensar la educación" (Vol. I), Política, educación y sociedad (Congreso internacional de didáctica), Ediciones Morata, Madrid, 1995.

13. Decimos algunas instituciones educativas porque muchas universidades no están desempeñando una de sus funciones fundamentales, como la investigación. Sólo se dedican a producir profesionales y no se pronuncian ni a favor ni en contra de las irracionalidades reinantes en la realidad social salvadoreña.

14. Tojeira, J. M. "La universidad y el servicio de la verdad en Centroamérica", Revista Realidad, No 54, pp. 735 y 736.

15. Cajiao, F. Poder y justicia en la escuela colombiano, Editores FES, Colombia, 1995 , p. 24.

16. Ibld., p. 26. (Las cursivas son mías) 